\title{
Fecundidade, dispersão e predação de sementes de Archontophoenix cunninghamiana H. Wendl. \& Drude, uma palmeira invasora da Mata Atlântica
}

\author{
ALEXANDER V. CHRISTIANINI ${ }^{1}$
}

(recebido: 15 de setembro de 2005; aceito: 28 de setembro de 2006)

\begin{abstract}
Fecundity, dispersal and predation of seeds of Archontophoenix cunninghamiana H. Wendl. \& Drude, an invasive palm in the Atlantic forest). Understanding the basic biology of invasive species can help us to choose the best management strategies to deal with the invasion. I report the phenology, seed production and dispersal of Archontophoenix cunninghamiana, an Australian palm that is invading Atlantic forest fragments. Field work was done in a 10 ha forest fragment

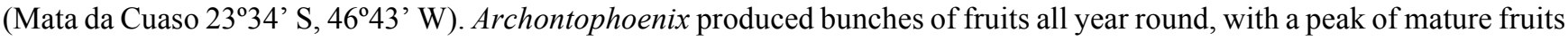
from October to February. Trees reach maturity around $18.5 \mathrm{~cm} \mathrm{DBH}$, each producing 4,119 $\pm 1,922$ seeds year $^{-1}$. Birds disperse the seeds, and nearly $15 \%$ of them escape post-dispersal seed predation during the time interval needed for germination. The spatial pattern of post-dispersal predation and the absence of pre-dispersal seed predation suggest a lack of specialized seed predators of Archontophoenix, as predicted by the enemy release hypothesis. Data obtained from this and other studies pointed out to a massive increase in seed production of $A$. cunninghamiana in a few years within the fragment. I suggest that this invasive palm can be taking advantage of the absence of Euterpe edulis Mart., a native palm which has similar biology, and was locally extinct due to human disturbances. Recommendations to control the invasion include the continuous removal of all Archontophoenix larger than $15 \mathrm{~cm} \mathrm{DBH}$, and the establishment of a buffer zone free of Archontophoenix around the fragment to decrease propagule pressure.
\end{abstract}

Key words - Atlantic forest, biological invasion, enemy release hypothesis, habitat fragmentation

RESUMO - (Fecundidade, dispersão e predação de sementes de Archontophoenix cunninghamiana H. Wendl. \& Drude, uma palmeira invasora da Mata Atlântica). Conhecer a biologia de espécies invasoras pode auxiliar na escolha de estratégias de manejo visando seu controle. Este estudo enfoca a fenologia, produção e dispersão de sementes de Archontophoenix cunninghamiana, uma palmeira nativa da Austrália que está invadindo fragmentos de Mata Atlântica. O estudo foi desenvolvido num fragmento florestal de 10 ha (Mata da Cuaso 23⒊' S, 46 $43^{\prime}$ 'W). Archontophoenix produziu frutos ao longo do ano, apresentando frutos maduros principalmente de outubro a fevereiro. As árvores atingem a maturidade com $18,5 \mathrm{~cm}$ DAP, cada uma produzindo $4.119 \pm 1.922$ sementes ano ${ }^{-1}$. Aves dispersam as sementes de Archontophoenix e $15 \%$ das sementes sobrevivem a predação pós-dispersão até o tempo requerido para germinação. O padrão espacial de predação pós-dispersão e a ausência de predadores de sementes pré-dispersão sugerem a ausência de predadores de sementes de Archontophoenix especializados, conforme predito pela hipótese de liberação de inimigos naturais. Dados deste e de outros estudos indicam um aumento expressivo na produção de sementes de A. cunninghamiana no fragmento em poucos anos. Archontophoenix pode estar se beneficiando da ausência de Euterpe edulis Mart., uma palmeira nativa que possui biologia similar e que foi extinta localmente devido à ação humana. Recomendações para controlar a invasão incluem a remoção contínua de todos Archontophoenix maiores que $15 \mathrm{~cm}$ DAP no fragmento e o estabelecimento de uma zona tampão ao redor do fragmento livre de Archontophoenix para dificultar a chegada de propágulos.

Palavras-chave - Floresta Atlântica, fragmentação de hábitat, hipótese de liberação de inimigos naturais, invasão biológica

\section{Introdução}

Plantas exóticas invasoras são freqüentemente capazes de modificar profundamente características

1. Programa de Pós-Graduação em Ecologia, Universidade Estadual de Campinas, Instituto de Biologia, Departamento de Zoologia, Caixa Postal 6109, 13083-970 Campinas, SP, Brasil. avchristianini@yahoo.com.br físicas e biológicas dos ambientes invadidos, seja pela exclusão competitiva de outras espécies, ou por alterações provocadas no solo, sombreamento, produtividade primária e suscetibilidade a fogo (D’Antonio \& Vitousek 1992, Vitousek et al. 1996). Acredita-se que as invasões biológicas estarão entre os cinco maiores eventos que irão alterar a biodiversidade global em todos os continentes e ecossistemas nos próximos 100 anos (Sala et al. 2000). 
Vários fatores parecem predispor certas espécies de plantas como invasoras, entre eles a produção e eficiência de dispersão de propágulos e interações competitivas com outras espécies, além de características do sítio invadido e eventos imprevisíveis, como pequenas perturbações naturais na vegetação (D’Antonio \& Vitousek 1992, Rejmánek \& Richardson 1996, Daehler 1998, Londsdale 1999, Davis et al. 2000). Espécies polinizadas por animais e/ou com frutos carnosos que atraem dispersores ocorrem acima do esperado como invasoras (Rejmanék \& Richardson 1996) já que os animais atuariam como facilitadores do processo de invasão. Perturbações antrópicas podem também facilitar processos de invasão por plantas exóticas. A remoção de indivíduos de uma comunidade aumenta temporariamente a disponibilidade local de recursos (como água, luz ou nutrientes no solo) que podem ser utilizados por outros indivíduos presentes no local (Davis et al. 2000). Caso haja propágulos presentes, espécies exóticas podem ter seu estabelecimento facilitado durante estes episódios de liberação temporária de recursos e menor competição (Davis et al. 2000). Uma vez estabelecidas, estas espécies exóticas podem aumentar suas populações e ampliar sua distribuição com o tempo, graças a suas propriedades competitivas, rápida propagação, resistência a fogo, etc (D'Antonio \& Vitousek 1992, Vitousek et al. 1996, Levine 2000), tornando-se invasoras.

Segundo a hipótese de liberação de inimigos naturais (Keane \& Crawley 2002), uma das razões para o sucesso de espécies exóticas como invasoras é que estas espécies encontram-se ocupando novos ambientes livres de seus inimigos naturais, como patógenos e herbívoros especializados, que controlam o crescimento de suas populações em sua área de distribuição original. Sem estes fatores de regulação, plantas invasoras podem investir em estruturas reprodutivas recursos que seriam utilizados em substâncias ou estruturas de defesa, além de alcançarem maior sobrevivência. Assim, uma proporção comparativamente maior dos propágulos produzidos pelas espécies invasoras poderia sobreviver em relação aos produzidos por espécies nativas, que continuam sob controle de seus inimigos naturais (Keane \& Crawley 2002). Por exemplo, patógenos e predadores de sementes especializados são reconhecidos como agentes de regulação de populações de várias espécies de plantas tropicais, promovendo grande mortalidade em áreas de maior concentração de propágulos, e.g. encontrados nas proximidades da planta-mãe (Janzen 1970). Insetos (e.g. Coleoptera, Lepidoptera,
Hymenoptera) cujas larvas se desenvolvem no interior de sementes freqüentemente dependem de uma ou poucas espécies de plantas para completarem seu ciclo de vida, caracterizando-se como predadores de sementes pré-dispersão especializados (Crawley 1992).

Com mais de $90 \%$ de sua área original convertidas para uso humano e com as áreas remanescentes em sua maior parte representadas por fragmentos pequenos e isolados, praticamente não há áreas de Mata Atlântica livres de algum tipo de impacto humano direto, como extrativismo, ou indireto, como efeitos de borda (Conservation International do Brasil et al. 2000). A proximidade de vários destes fragmentos a áreas urbanas densamente povoadas predispõe um contato maior destes remanescentes com espécies exóticas cultivadas para fins agrícolas ou ornamentais, as quais podem potencialmente colonizá-los. Dentre estas espécies, Archontophoenix cunninghamiana H. Wendl. \& Drude é uma palmeira exótica nativa da Austrália que tem invadido fragmentos de Mata Atlântica (Dislich et al. 2002). Aves dispersam as sementes de Archontophoenix em sua área de distribuição original (Zona \& Henderson 1989), mas pouco se sabe sobre a dispersão e sobrevivência pósdispersão das sementes nas áreas invadidas (Hasui \& Höfling 1998). Por influenciar todos os processos subseqüentes relacionados à demografia de populações vegetais, estudos sobre a dispersão de sementes de plantas invasoras podem auxiliar na compreensão dos mecanismos que atuam no processo de invasão e na elaboração de propostas de controle destas espécies.

Este trabalho foi conduzido em um fragmento de Mata Atlântica sob invasão por Archontophoenix, e em seus arredores, buscando aumentar o conhecimento sobre o processo de invasão para permitir a elaboração de futuras estratégias de manejo da espécie. Os objetivos deste estudo incluem: 1) caracterizar o padrão fenológico e a produção de propágulos da espécie; 2) estabelecer o tamanho mínimo a partir do qual os indivíduos tornam-se adultos, confrontando os valores obtidos com a informação disponível na literatura (e.g. Dislich et al. 2002); 3) caracterizar os animais que atuam como dispersores e a eficiência de remoção dos propágulos da planta-mãe; 4) testar a validade da hipótese de liberação de inimigos naturais, verificando a ocorrência de predação pré-dispersão por predadores especializados, bem como a variação espacial na predação pós-dispersão de sementes de Archontophoenix. Se Archontophoenix está se beneficiando da dispersão de sementes por animais espera-se uma grande eficiência na remoção de 
propágulos (e.g. maior parte dos frutos removidos em poucos dias). Caso a hipótese de liberação de inimigos naturais seja verdadeira para a invasão de fragmentos florestais por Archontophoenix, espera-se que a predação de sementes pré-dispersão por insetos que se desenvolvem no interior da semente seja pouco expressiva, e que a predação de sementes pós-dispersão não esteja concentrada nas imediações da planta-mãe, dada a ausência de predadores de sementes especializados.

\section{Material e métodos}

Área de estudo - Este estudo foi desenvolvido em um fragmento florestal urbano, a Reserva da Cidade Universitária Armando de Salles Oliveira (Mata da Cuaso: 23⒊' S, $46^{\circ} 43^{\prime}$ W). A Mata da Cuaso ocupa uma área de $c a .10$ ha em uma depressão do terreno (Rossi 1994). Apesar de ter sofrido alterações no passado, a Mata da Cuaso representa uma amostra da vegetação original de Mata Atlântica que cobria a região da cidade de São Paulo. Atualmente, o processo de sucessão da vegetação da Mata da Cuaso encontra-se limitado devido ao seu isolamento, permitindo apenas a chegada de diásporos de áreas perturbadas do entorno, além dos indivíduos que ocorrem no fragmento (Dislich \& Pivello 2002). Embora a área seja protegida, estando cercada desde 1979, pelo menos 30 espécies vegetais exóticas podem ser encontradas ao lado de espécies nativas (Rossi 1994). Entre as espécies nativas que se destacam no estrato arbóreo estão Alchornea sidifolia Müll. Arg., A triplinervia (Spreng.) Müll. Arg., Cedrela fissilis Vell., Ficus insipida Willd., Piptadenia gonoacantha (Mart.) J. F. Macbr. e Machaerium nyctitans (Vell.) Benth. (Rossi 1994, Dislich \& Pivello 2002). A temperatura média anual é $19,2^{\circ} \mathrm{C}$, com pluviosidade média anual de $1.207 \mathrm{~mm}$, sendo os meses de maio a setembro a estação de menor pluviosidade (Gorrezio-Roizman 1993).

Espécie de estudo - Archontophoenix cunninghamiana $\mathrm{H}$. Wendl. \& Drude é uma palmeira arborescente de origem australiana, conhecida popularmente como palmeira-seafórtia. Espécie monóica, tolerante a climas temperados, propaga-se apenas por sementes e tem sido largamente cultivada com fins paisagísticos em parques e jardins públicos ou privados (Lorenzi 1996). Possui crescimento monopodial e frutificação abundante, apresentando frutos esféricos vermelhos, amplamente consumidos por aves em seu habitat original (Waterhouse \& Quinn 1978, Zona \& Henderson 1989). Os frutos de A. cunninghamiana medem (comprimento $\times$ diâmetro) $12,7 \pm 0,8 \times 11,6 \pm 0,9 \mathrm{~mm}$, pesando $1,1 \pm 0,2 \mathrm{~g}$

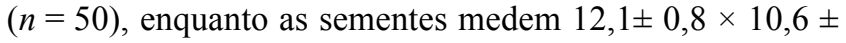
$0,7 \mathrm{~mm}$ e pesam $0,9 \pm 0,2 \mathrm{~g}(n=50)$.

Métodos - A fenologia de A.cunninghamiana foi determinada a partir da observação de 40 indivíduos marcados na Mata da Cuaso e no entorno, nos jardins do Instituto de Biociências da Universidade de São Paulo. Mensalmente, de julho de
2000 a junho de 2001, os indivíduos foram observados com binóculo $8 \times 40$ quanto à presença e número de cachos contendo botões, flores e frutos (imaturos ou maduros). Paralelamente, 26 indivíduos foram acompanhados quanto à presença ou ausência de estruturas reprodutivas (cachos), de forma a estabelecer relações entre o tamanho e a reprodução nestas plantas. Uma estimativa da produção de propágulos cacho ${ }^{-1}$ foi obtida a partir da coleta de um cacho contendo frutos maduros em três indivíduos distintos. Os cachos foram escolhidos com base na acessibilidade das plantas, pela representatividade de tamanhos dos cachos em relação à produção natural da população estudada, e por seu estágio de maturação, de forma a reduzir perdas de propágulos anteriores à coleta devido à ação de animais dispersores. Quinhentos frutos coletados tiveram a polpa removida e foram inspecionados visualmente para localizar aberturas na testa da semente que denunciassem a perfuração por larvas de insetos predadores de sementes pré-dispersão (Pizo 1997).

A caracterização da fauna de dispersores de propágulos de Archontophoenix foi determinada a partir de observações focais de indivíduos reprodutivos contendo cachos maduros. Treze sessões de observação de 50 a 180 minutos cada foram realizadas durante a manhã (entre $7 \mathrm{~h} 00$ e $11 \mathrm{~h} 00$ ) e a tarde (entre $17 \mathrm{~h} 00$ e 18h00) em cinco locais diferentes, na Mata da Cuaso e arredores, num total de 21 horas de observação. Durante as observações foram anotadas as espécies de aves visitantes (Höfling \& Camargo 1996), o número de indivíduos, o comportamento de forrageio (se engoliam ou derrubavam os frutos) e o número de frutos removidos.

O impacto de predadores de sementes pós-dispersão foi verificado a partir de um experimento executado na Mata da Cuaso com sementes despolpadas, imitando sementes regurgitadas por aves. Três sementes de $A$. cunninghamiana foram colocadas em estações de remoção dispostas à distâncias fixas da planta-mãe $(1,5,10,15$ e 20 m). As estações de remoção foram estabelecidas ao longo de quatro transecções equidistantes e a $90^{\circ}$ entre sí, iniciadas na base do tronco de uma palmeira adulta de Archontophoenix. O experimento foi replicado em quatro plantas adultas de A. cunninghamiana, num total de 80 estações de remoção (20 estações de remoção por planta). A presença e o estado das sementes (viva ou predada) foram verificadas a 10, 20, 49 e 80 dias após o início do experimento. A partir de 75 dias as sementes iniciam a germinação (Lorenzi 1996). A predação de sementes pós-dispersão por insetos foi verificada ao longo deste mesmo experimento por meio da inspeção visual das sementes, em busca de perfurações deixadas por larvas de insetos (Pizo 1997).

Análises estatísticas - Relações entre tamanho da palmeira e reprodução foram investigadas com o uso de regressão logística (Trexler \& Travis 1993), utilizando o diâmetro à altura do peito (DAP) como variável explanatória e reprodução (produzindo cachos ou não) como variável resposta. A regressão logística estima os parâmetros da regressão assumindo uma distribuição binomial da variável dependente 
frente a variações nas variáveis independentes. Ela gera uma curva de probabilidade de transição entre estágios da variável categórica que mais se ajusta aos dados, testando o ajuste do modelo por máxima verossimilhança (Trexler \& Travis 1993). Uma das vantagens de se utilizar a regressão logística é que ela permite assinalar a probabilidade de ocorrência de um determinado estado da variável dependente (no caso, produzir cachos) para um dado valor das variáveis independentes (no caso, DAP). A sobrevivência das sementes dispostas nas estações de remoção a distâncias variáveis da planta-mãe foi comparada por análise de sobrevivência (teste de Peto \& Peto, Pyke \& Thompson 1986). Ao longo do texto, as médias são sempre apresentadas seguidas do respectivo desvio padrão.

\section{Resultados}

Archontophoenix cunninghamiana H. Wendl. \& Drude apresenta estruturas reprodutivas em diferentes estágios ao longo de todo o ano, produzindo frutos maduros de julho a março, com um pico de frutificação entre outubro e fevereiro (figura 1). A reprodução de Archontophoenix é fortemente influenciada pelo tamanho (DAP) da planta (regressão logística: $\mathrm{y}=\exp (-20,9+1,1 \times) / 1+\exp (-20,9+1,1 \times) ; \chi^{2}=32,0$ $g l=1 ; P<0,001)$. Um indivíduo de apenas $18,6 \mathrm{~cm}$ DAP foi observado em reprodução no período, sendo este o tamanho mínimo observado para que os indivíduos atinjam a maturidade, mas a probabilidade de produzir cachos aumenta abruptamente, para 95\%, em indivíduos com 21,6 cm DAP. Indivíduos adultos produzem em média 3,0 $\pm 1,4$ cachos de frutos $a^{-1}(n=29$ plantas $)$, com cada cacho contendo em média $1.373 \pm 687$ frutos $(n=3)$. Como cada fruto contém apenas uma semente, cada indivíduo adulto de Archontophoenix produz potencialmente $4.119 \pm 1.922$ sementes ano ${ }^{-1}$.

Seis espécies de aves de pequeno porte foram observadas consumindo os frutos de Archontophoenix, num total de 69 visitas, perfazendo 3,3 visitas hora-1 (tabela 1). As sementes engolidas por Pitangus sulphuratus (Linnaeus, 1766), Turdus rufiventris Vieillot, 1818 e T. leucomelas Vieillot, 1818 são regurgitadas intactas poucos minutos após o consumo dos frutos (observação pessoal) e, assim, estas espécies podem ser consideradas como dispersores legítimos das sementes. Assumindo um número médio constante de visitas de aves e remoção ou derrubada de frutos ao longo do dia (12 horas dia ${ }^{-1}$ ), as aves necessitariam de apenas 13 dias para remover todos os frutos de um cacho (8,6 frutos removidos e derrubados hora ${ }^{-1}$, tabela 1). Durante o período de frutificação de Archontophoenix uma grande quantidade de sementes regurgitadas pode ser encontrada sobre o folhiço na Mata da Cuaso ou sob as árvores dos jardins do Departamento de Botânica (observação pessoal) distante cerca de 80 metros do fragmento, indicando que as aves estão transportando as sementes desta palmeira de forma eficiente e a distâncias consideráveis dos indivíduos adultos.

Não foram observadas evidências de predação de sementes pré-dispersão em uma amostra de 500 frutos coletados diretamente dos cachos. No entanto, 84,2\% das sementes dispostas nos experimentos de predação pós-dispersão foram predadas (destruídas no local ou removidas) em até 80 dias. Embora tenham sido observadas diferenças na proporção de sementes que são predadas a diferentes distâncias da planta-mãe (análise de sobrevivência: $\chi^{2}=23,4 ; g l=4 ; P<0.001$ ), não há um padrão consistente de predação em relação à distância da planta-mãe, com os maiores percentuais de sementes sobreviventes a 1 e a 15 metros da palmeira (figura 2). Cerca de 15\% das sementes sobrevivem pelo menos até o tempo mínimo necessário para iniciarem a germinação (75 dias). Besouros Coccotrypes

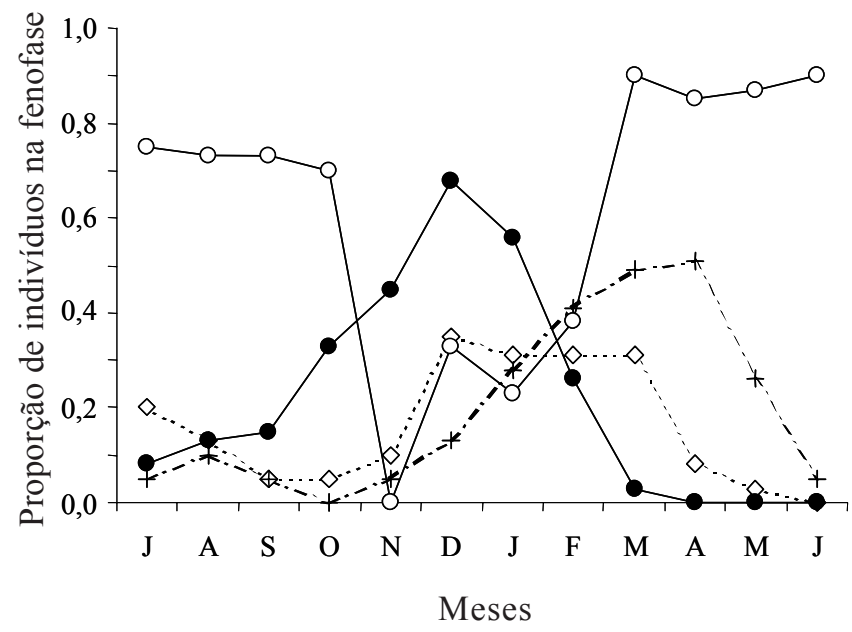

Figura 1. Proporção dos indivíduos de Archontophoenix cunninghamiana $\mathrm{H}$. Wendl. \& Drude $(n=40)$ em diferentes fenofases na Mata da Cuaso e arredores, entre julho de 2000 e junho de 2001. Categorias das fenofases: $\cdots \diamond \cdots=$ botões; $\ldots+\ldots=$ flores $;-0-=$ frutos imaturos; $-\bullet-=$ frutos maduros. Um mesmo indivíduo pode apresentar mais de uma fenofase, de acordo com o número e a maturação dos cachos presentes.

Figure 1. Proportion of individuals of Archontophoenix cunninghamiana $\mathrm{H}$. Wendl. \& Drude $(n=40)$ showing different phenophases within the Mata da Cuaso and surroundings, between July 2000 and June 2001. Categories of phenophases: $\ldots \diamond \ldots=$ buds; $\ldots+\ldots=$ flowers $;-0-=$ unripe fruits; $-\bullet-=$ ripe fruits. Each plant may show more than one phenophase, following the number and maturity of bunches present at a time. 
Tabela 1. Espécies de aves observadas consumindo frutos de Archontophoenix cunninghamiana H. Wendl. \& Drude na Mata da Cuaso e arredores, comportamento frente aos frutos e respectivo número de visitas ao longo de 21 horas de observações focais. Os valores indicados para número de aves referem-se a médias visita ${ }^{-1}$, enquanto o número de frutos removidos e número de frutos derrubados referem-se a médias ave visita ${ }^{-1}$. ( $\mathrm{DS}=$ dispersor de sementes, engole ou carrega o fruto para consumo em outra árvore; $\mathrm{CP}$ = consumidor de polpa, consome a polpa no local e derruba a semente sob a planta-mãe). As espécies estão arranjadas em ordem decrescente de número de visitas de acordo com o comportamento de forrageio. Filiação taxonômica segundo Sick (1997).

Table 1. Bird species observed removing fruits from Archontophoenix cunninghamiana $\mathrm{H}$. Wendl. \& Drude within the Mata da Cuaso and surroundings, fruit eating behavior and number of visits for 21 hours of focal tree observations. Values for the number of birds per visit are means visit ${ }^{-1}$, while the number of fruits removed and number of fruits dropped are means bird visit ${ }^{-1}$. (DS = seed disperser, swallow the seed or remove the fruit for later consumption in other tree; $\mathrm{CP}=$ pulp predator, feed on fruit pulp and drops the seed under the mother tree). Bird species are presented following the number of visits within a fruit eating behavior category. Bird taxonomy follows Sick (1997).

\begin{tabular}{llccccc}
\hline Família & Espécie & Frugívoro & Número \\
tipo & de visitas & $\begin{array}{c}\text { Aves } \\
\text { por visita }\end{array}$ & Número de frutos \\
\cline { 5 - 7 } & & DS & 36 & 1,0 & 1,0 & 0 \\
\hline Tyrannidae & Pitangus sulphuratus (Linnaeus, 1766) & DS & 26 & 1,1 & 1,4 & 0,4 \\
Muscicapidae & Turdus rufiventris Vieillot, 1818 & DS & 1 & 2,0 & 1,0 & 3,0 \\
& T. leucomelas Vieillot, 1818 & CP & 3 & 2,0 & 0,2 & 13,2 \\
Psittacidae & Brotogeris chiriri (Vieillot, 1818) & CP & 2 & 2,0 & 0 & 1,0 \\
Emberizidae & Thraupis sayaca (Linnaeus, 1766) & CP & 1 & 1,0 & 0 & 1,0 \\
& Saltator similis d'Orbigny \& Lafresnaye, 1837 & CP & & & \\
\end{tabular}

circumdata Fonseca, 1930 (Scolytinae, Coleoptera), predadores generalistas de sementes (S.A. Vanin, comunicação pessoal), foram responsáveis por pelo menos $48,3 \%$ das sementes predadas, sendo o restante provavelmente predado por roedores.

\section{Discussão}

O presente estudo indica que frutos maduros de Archontophoenix cunninghamiana H. Wendl. \& Drude estão disponíveis ao longo de quase todo o ano, são removidos e dispersos por aves comuns a ambientes perturbados (Sick 1997) em poucos dias e que cerca de $15 \%$ destas sementes sobrevivem o tempo mínimo necessário para germinação. Assim, a dispersão de sementes não constitui um obstáculo a colonização de fragmentos florestais por esta espécie.

Aves certamente estão contribuindo para o processo de invasão ao dispersarem sementes desta palmeira para o interior da mata. Ao se deslocarem entre áreas externas e internas do fragmento, animais podem atuar como vetores de dispersão de sementes de espécies vegetais exóticas, facilitando e amplificando o processo de invasão (Janzen 1983). A área de uso destas aves irá determinar a que distância máxima as sementes podem ser dispersas. Embora as espécies observadas sejam de pequeno porte, há informações sobre a dispersão de Archontophoenix por tucanos-de-bicoverde (Ramphastos dicolorus Linnaeus, 1766) (Hasui \& Höfling 1998), que visitam a área de estudo nos

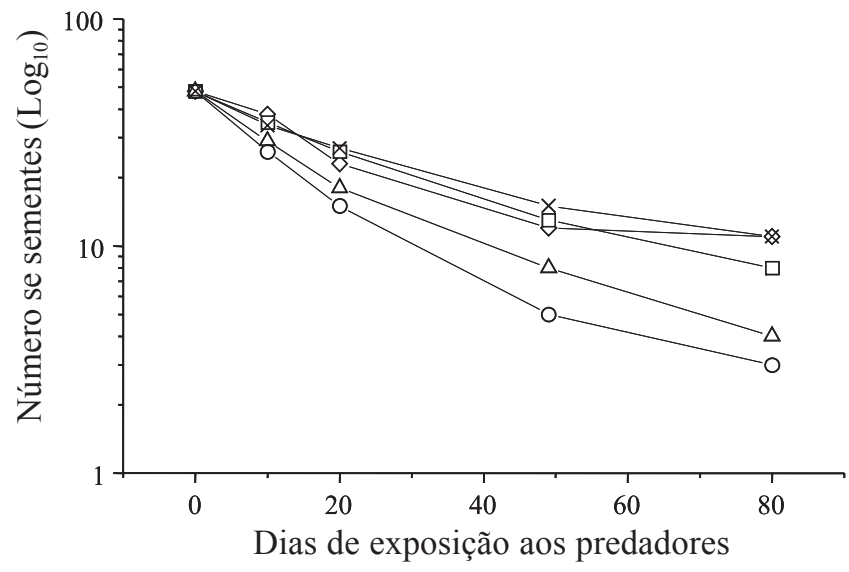

Figura 2. Sobrevivência pós-dispersão das sementes de Archontophoenix cunninghamiana $\mathrm{H}$. Wendl. \& Drude em relação à distância da planta-mãe. Classes de distância: $-\diamond-=$ $1 \mathrm{~m} ;-\square-=5 \mathrm{~m} ;-\chi_{-}=10 \mathrm{~m} ;-\triangle-=15 \mathrm{~m} ;-0-=20 \mathrm{~m}$.

Figure 2. Post-dispersal seed survival of Archontophoenix cunninghamiana $\mathrm{H}$. Wendl. \& Drude at different distances from the mother tree. Distance classes: $-\diamond-=1 \mathrm{~m} ;-\square-=5 \mathrm{~m}$; $-{ }_{-}=10 \mathrm{~m} ;-\triangle_{-}=15 \mathrm{~m} ;-\mathrm{O}_{-}=20 \mathrm{~m}$. 
períodos de frutificação desta palmeira (Höfling \& Camargo 1996), o que possibilitaria a dispersão de sementes a distâncias muito maiores.

Como não houve um padrão espacial consistente na predação de sementes com o aumento da distância da planta-mãe para Archontophoenix e não foi observada predação pré-dispersão, predadores generalistas devem ser os principais responsáveis pela predação de sementes desta palmeira, conforme o esperado pela hipótese de liberação de inimigos naturais (Keane \& Crawley 2002). Para se ter uma idéia da vantagem comparativa que pode ser proporcionada pela ausência de predadores de sementes especializados, vale indicar que larvas do besouro Revena rubiginosa Boh., 1836 (Curculionidae), que se desenvolvem somente no interior das sementes de Syagrus romanzoffiana (Cham.) Glassman, predam entre $42 \%$ e 59\% das sementes desta espécie em florestas semidecíduas de São Paulo (Alves-Costa 2004). Estudos que comparem os níveis de predação pré- e pós-dispersão das sementes de Archontophoenix e de espécies de palmeiras nativas ao longo das áreas invadidas podem fornecer novas evidências sobre a validade e generalização da hipótese de liberação de inimigos naturais para o processo de invasão por Archontophoenix.

Mesmo apresentando uma mortalidade de sementes relativamente alta, Archontophoenix está invadindo o fragmento com bastante sucesso. Se o número de sementes que são dispersas até o fragmento compensa a alta mortalidade ou satura os microhábitats disponíveis para recrutamento, a predação de sementes não se constitui numa barreira eficiente para a regulação das populações desta palmeira (Keane \& Crawley 2002). De fato, Archontophoenix tem apresentado recrutamento em níveis bastante superiores ao das espécies arbóreas nativas mais comuns (Dislich \& Pivello 2002, Dislich et al. 2002).

O tamanho mínimo encontrado para indivíduos reprodutivos de Archontophoenix neste estudo $(\mathrm{DAP}=18,6 \mathrm{~cm})$ foi consideravelmente inferior ao encontrado por Dislich et al. (2002) (DAP $=25 \mathrm{~cm})$ na mesma área. A discrepância entre estes resultados pode ser devida ao acompanhamento da fenologia desta espécie ao longo de um ano neste estudo, além de variações individuais e estacionais dos indivíduos amostrados. Utilizando-se das informações sobre crescimento diamétrico de Archontophoenix apresentada em Dislich et al. (2002) é possível indicar que indivíduos com $10 \mathrm{~cm}$ de DAP necessitam de cerca de 7 anos para atingir o tamanho mínimo reprodutivo $(\mathrm{DAP}=18,6 \mathrm{~cm}$ ) ou 12 anos para atingir $21,6 \mathrm{~cm}$ DAP, tamanho em que detêm $95 \%$ de probabilidade de tornarem-se indivíduos reprodutivos. Como o número de indivíduos de Archontophoenix com DAP $\geq 9,5 \mathrm{~cm}$ é bastante expressivo no interior da Mata da Cuaso (Dislich \& Pivello 2002, Dislich et al. 2002), é esperado um crescimento ainda maior na população desta palmeira invasora com a maturidade destes indivíduos. A lentidão de medidas que contenham o processo de invasão por esta palmeira pode trazer impactos severos à comunidade local de plantas, mesmo se considerarmos que Archontophoenix compete com as espécies nativas apenas por espaço. Utilizando os dados de densidade, crescimento e mortalidade de Archontophoenix fornecidos em Dislich et al. (2002) e o DAP indicativo de maturidade obtido neste estudo, é possível verificar que dos 305 indivíduos com DAP $\geq 9,5 \mathrm{~cm}$ presentes numa área de 2,1 ha no interior da mata (área 2 no referido trabalho) cerca de 277 indivíduos devem atingir $95 \%$ de probabilidade (DAP $\geq 21,6 \mathrm{~cm}$ ) de tornarem-se adultos nos próximos anos caso as condições observadas por Dislich et al. (2002) se mantenham. Esta é uma estimativa conservadora, já que vários destes indivíduos apresentam DAP superior a 9,5 cm, e devem atingir a maturidade antes de doze anos decorridos do estudo de Dislich et al. (2002). Assim, a produção local de sementes de Archontophoenix deverá sofrer um grande aumento em pouco tempo. A partir de extrapolações do número médio de sementes produzidas planta $a^{-1}{ }^{-1}$, espera-se uma produção anual de mais de 1.140.000 sementes somente nesta área.

Embora o sucesso de Archontophoenix como invasora esteja ligado a características biológicas da planta, particularidades locais como a abundância de indivíduos adultos no entorno, a eficiência de dispersão de propágulos e perturbações antrópicas mais severas, como corte seletivo que ocorreu no passado na Mata da Cuaso (Rossi 1994), podem estar beneficiando esta palmeira exótica. Palmeiras arbóreas nativas são especialmente pouco representadas no local, com poucos indivíduos de Syagrus romanzoffiana (Cham.) Glassman e a ausência do palmito Euterpe edulis Mart., de ocorrência esperada no local, mas provavelmente extinto por corte seletivo. É possível que Archontophoenix tenha sobreposição parcial com o nicho de Euterpe, e se beneficiou da extinção local desta espécie. Ambas espécies compartilham características arquiteturais (Waterhouse \& Quinn 1978, Alves et al. 2004), são muito abundantes no interior de florestas conservadas nas suas respectivas áreas de distribuição originais (Waterhouse \& Quinn 1978, Reis et al. 2000), possuem frutos e sementes de tamanhos semelhantes 
(observação pessoal) e são dispersas predominantemente por aves (Zona \& Henderson 1989, Galetti et al. 1999). Embora uma relação de causa e efeito entre a ausência de Euterpe e a invasão de Archontophoenix seja de difícil comprovação, ela fornece uma perspectiva útil para o entendimento do processo de invasão e a elaboração de propostas de manejo e controle da palmeira invasora.

Medidas de controle de Archontophoenix devem contemplar a remoção dos indivíduos adultos desta espécie não apenas no interior da Mata da Cuaso, mas também em seus arredores, para dificultar a chegada de propágulos até o fragmento. Como as aves que atuam como dispersores de sementes são predominantemente pequenas e de hábitos generalistas, deslocando-se freqüentemente entre o interior da mata e áreas externas (Höfling \& Camargo 1996, observação pessoal), uma zona tampão ao redor da mata, livre de Archontophoenix, deve ser implementada para dificultar a chegada de propágulos. A largura ideal desta zona tampão deverá ser estabelecida a partir de observações do deslocamento das aves no local. A substituição gradual de Archontophoenix por palmeiras nativas de potencial ornamental (como Euterpe edulis e Syagrus romanzoffiana) nos arredores da mata e no restante do câmpus também contribuiria neste sentido. Contudo, a retirada de plântulas e indivíduos jovens de Archontophoenix irá acarretar um esforço expressivamente maior, devido ao grande número de indivíduos presentes em praticamente toda a mata (Dislich et al. 2002). A retirada de indivíduos jovens próximos da maturidade (e.g. DAP $\geq 15 \mathrm{~cm}$ ) a intervalos regulares de dois anos poderia ser empregada como alternativa adicional de manejo, eliminando gradativamente a espécie do fragmento e permitindo o monitoramento do processo de invasão. Paralelamente, um programa de reintrodução de Euterpe edulis na Mata da Cuaso poderia repor indivíduos desta espécie e substituir Archontophoenix como recurso alimentar das aves. O estado atual de degradação da Mata Atlântica impõe a necessidade de manejo não apenas dos fragmentos remanescentes, mas também das áreas de entorno destes fragmentos. O controle de espécies exóticas invasoras deve fazer parte destas estratégias.

Agradecimentos - Ao Dr. Sérgio A. Vanin, que gentilmente identificou os besouros predadores das sementes de Archontophoenix; à Dra. Marico Meguro, pela autorização de estudo na área da Mata da Cuaso; ao Ricardo Dislich, pelas discussões interessantes sobre o processo de invasão; à Flávia T. Colpas, Erica Hasui e a um revisor anônimo, que enriqueceram o manuscrito com suas críticas. Sou grato ainda ao CNPq, pelo suporte financeiro durante a coleta de dados, ao Programa de Pós-Graduação em Ecologia da Unicamp e a uma bolsa de doutoramento da Fapesp, que forneceram suporte logístico e financeiro, respectivamente, durante a redação deste manuscrito.

\section{Referências bibliográficas}

ALVES, L.F., MARTINS, F.R. \& SANTOS, F.A.M. 2004. Allometry of a neotropical palm, Euterpe edulis Mart. Acta Botanica Brasilica 18:369-374.

ALVES-COSTA, C.P. 2004. Efeitos da defaunação de mamíferos herbívoros na comunidade vegetal. Tese de doutorado, Universidade Estadual de Campinas, Campinas.

CONSERVATION INTERNATIONAL DO BRASIL, FUNDAÇÃO SOS MATAATLÂNTICA, FUNDAÇÃO BIODIVERSITAS, INSTITUTO DE PESQUISAS ECOLÓGICAS, SECRETARIADOMEIOAMBIENTEDO ESTADO DE SÃO PAULO, SEMAD/INSTITUTO ESTADUAL DE FLORESTAS - MG. 2000. Avaliação e ações prioritárias para a conservação da Biodiversidade da Mata Atlântica e Campos Sulinos. Ministério do Meio Ambiente/Secretaria de Biodiversidade e Florestas, Brasília.

CRAWLEY, M.J. 1992. Seed predators and plant population dynamics. In Seeds: The Ecology of Regeneration in Plant Communities (M. Fenner, ed.). CABI, Wallingford, p.157-191.

D'ANTONIO, C.M. \& VITOUSEK, P.M. 1992. Biological invasions by exotic grasses, the grass/fire cycle and global change. Annual Review of Ecology and Systematics 23:63-87.

DAEHLER, C.C. 1998. The taxonomic distribution of invasive Angiosperm plants: ecological insights and comparison to agricultural weeds. Biological Conservation 84:167-180.

DAVIS, M.A., GRIME, J.P. \& THOMPSON, K. 2000. Fluctuating resources in plant communities: a general theory of invasibility. Journal of Ecology 88:528-534.

DISLICH, R. \& PIVELLO V.R. 2002. Tree structure and species composition changes in an urban tropical forest fragment (São Paulo, Brazil) during a five-year period. Boletim de Botânica da Universidade de São Paulo 20:1-12

DISLICH, R., KISSER, N. \& PIVELLO, V.R. 2002. Ainvasão de um fragmento florestal em São Paulo (SP) pela palmeira australiana Archontophoenix cunninghamiana $\mathrm{H}$. Wendl. \& Drude. Revista Brasileira de Botânica 25:55-64.

GALETTI, M., ZIPARRO, V.B. \& MORELLATO, P.C. 1999. Fruiting phenology and frugivory on the palm Euterpe edulis in a lowland Atlantic forest of Brazil. Ecotropica 5:115-122.

GORREZIO-ROIZMAN, L. 1993. Fitossociologia e dinâmica do banco de sementes de populações arbóreas de floresta secundária em São Paulo, SP. Tese de doutorado, Universidade de São Paulo, São Paulo. 
HASUI, E. \& HÖFLING, E. 1998. Preferência alimentar de aves frugívoras de um fragmento de floresta estacional semidecídua secundária, São Paulo, Brasil. Iheringia, Série Zoologia 84:43-64.

HÖFLING, E. \& CAMARGO, H.F.A. 1996. Aves no Campus: Cidade Universitária Armando de Salles Oliveira. Edusp \& IB-USP, São Paulo.

JANZEN, D.H. 1970. Herbivores and the number of tree species in tropical forests. The American Naturalist 104:501-528.

JANZEN, D.H. 1983. No park is an island: increase in interference from outside as park size decrease. Oikos 41:402-410.

KEANE, R.M. \& CRAWLEY, M.J. 2002. Exotic plant invasions and the enemy release hypothesis. Trends in Ecology and Evolution 17:164-170.

LEVINE, J.M. 2000. Species diversity and biological invasions: relating local process to community pattern. Science 288:852-854.

LONDSDALE, W.M. 1999. Global patterns of plant invasions and the concept of invasibility. Ecology 80:1522-1536.

LORENZI, H. 1996. Palmeiras no Brasil - nativas e exóticas. Editora Plantarum, Nova Odessa.

PIZO, M.A. 1997. Seed dispersal and predation in two populations of Cabralea canjerana (Meliaceae) in the Atlantic Forest of southeastern Brazil. Journal of Tropical Ecology 13:559-578.

PYKE, D.A. \& THOMPSON, J.N. 1986. Statistical analysis of survival and removal rate experiments. Ecology 67:240-245.
REIS, M.S., FANTINI,A.C., NODARI, R.O., REIS,A., GUERRA M.P. \& MANTOVANI, A. 2000. Management and conservation of natural populations in Atlantic rain forest: the case study of palm heart (Euterpe edulis Martius). Biotropica 32:894-902.

REJMÁNEK, M.\& RICHARDSON, D.M. 1996. What atributes make some plant species more invasive? Ecology 77:1655-1661.

ROSSI, L. 1994. A flora arbóreo-arbustiva da mata da reserva da cidade universitária "Armando de Salles Oliveira" (São Paulo, Brasil). Boletim do Instituto de Botânica de São Paulo 9:1-105.

SALA, O.E., CHAPIN III, F.S., ARMESTO, J.J., BERLOW, E., BLOOMFIELD, J., DIRZO, R., HUBER-SANWALD, E., HUENNEKE,L.F., JACKSON, R.B., KINZIG,A., LEEMANS, R., LODGE, D.M., MOONEY, H.A., OESTERHELD, M., POFF, N.L., SKYES, M.T., WALKER, B.H., WALKER, M. \& WALL, D.H. 2000. Global biodiversity scenarios for the year 2100. Science 287:1770-1774.

SICK, H. 1997. Ornitologia Brasileira. Nova Fronteira, Rio de Janeiro.

TREXLER, J.C. \& TRAVIS, J. 1993. Nontraditional regression analyses. Ecology 74:1629-1637.

VITOUSEK, P.M., D’ANTONIO, C., LOOPE, L.L. \& WESTBROOKS, R. 1996. Biological invasions as global environmental change. American Scientist 84:468-478.

WATERHOUSE, J.T. \& QUINN, C.J. 1978. Growth patterns in the stem of the palm Archontophoenix cunnighamiana. Botanical Journal of the Linnean Society 77:73-93.

ZONA, S. \& HENDERSON, A. 1989. A review of animalmediated seed dispersal of palms. Selbyana 11:6-21. 